\title{
International Campaign for Cures of Spinal Cord Injury Paralysis (ICCP): another step forward for spinal cord injury research
}

\author{
M Adams ${ }^{1}$ and JFR Cavanagh ${ }^{*}, 1$ \\ International Spinal Research Trust, Bramley Business Centre, Bramley, Guildford, Surrey, UK
}

For over 20 years, charitable organizations have worked to promote research that will cure the paralysis associated with spinal cord injury (SCI). When they began this quest, the consequences of SCI were thought to be permanent; that once damaged, the spinal cord could not be repaired. Today, the same organizations are credited with funding research that has realized many significant advances, brought new optimism and changed the outlook of researchers, clinicians and injured individuals alike. Progress in understanding the basic biology of spinal cord repair means that it is now a case of how soon useful treatments will be available, rather than if there will ever be anything to offer. With this in mind, many of the organizations that promote spinal cord research have formed an alliance to determine the ways in which their collaboration can hasten progress. The mission and objectives of this alliance, termed the International Campaign for Cures of Spinal Cord Injury Paralysis (ICCP), are described here.

Spinal Cord (2004) 42, 273-280. doi:10.1038/sj.sc.3101597; Published online 17 February 2004

Keywords: spinal cord injury; spinal cord repair; ICCP; nonprofit-funding organizations

\section{Introduction}

It is estimated that, every year, up to 1000 people in the UK, and up to 100,000 worldwide, survive a spinal cord injury (SCI) that leaves them paralysed, wheelchairbound and, in many cases, reliant on the support of carers (www.campaignforcure.org). With an average age of injury of 33.4 years and near normal life expectancy, there is a steady increase in the number of people affected, and it is estimated that around 2.5 million people will be living with SCI by 2005. As a result, the economic cost of long-term care and social provision for this group is in excess of tens of billons of dollars annually (www.campaignforcure.org). Reliable reports estimate that over $£ 500$ million is spent each year in the UK alone, and in the US the annual cost is in the order of US\$7.7 billion.

Until recently, the possibility of developing effective treatments to restore function following traumatic SCI seemed bleak. In the past 10 years, however, scientific breakthroughs in animal models raise the expectation that treatments that will restore some worthwhile function to human patients will be available in the near future. Many of the basic and clinical studies that have gone into developing these treatments rely on funding provided by private, charitable foundations that are

*Correspondence: JFR Cavanagh, International Spinal Research Trust, 8a Bramley Business Centre, Station Road, Bramley, Guildford, Surrey GU5 0AZ, UK dedicated to developing useful treatments for this condition. The unflagging drive and optimism of these organizations has provided both the means and the inspiration for recent experimental successes, and helped to dispel the notion that talk of successful treatment could only raise false hope. Clearly, the more we understand about the uninjured spinal cord and the changes that accompany SCI, the better we can target therapies to limit damage after injury and restore function. As new discoveries are made, and as the expectations of success change, so too do the roles of these foundations.

\section{Roles of private foundations}

Many of the private foundations that are dedicated to ensuring that treatments for SCI are developed were founded by individuals who have experienced SCI either themselves or in someone close to them. Historically, these organizations have raised income from a number of sources (including Government, corporate and private) and, with careful planning, have redistributed this to support research according to the defined objectives of each organization. As with all funding organizations, sources of income must be disclosed and the systems of allocating this must be open and transparent. This includes using recognized peer-review processes to ensure that only high-quality research 
studies are funded, which are accepted as meaningful and scientifically sound by the scientific and medical communities.

\section{Determining research priorities}

The prime objective of all funding bodies is to provide the most effective support for relevant research. At the same time, through close links with injured individuals, private foundations can articulate the issues that are most important to those living with SCI. Identifying research targets based on the needs of both research and SCI communities increases the legitimacy of these targets and enables funds to be directed specifically to relevant research projects. Collaboration between private charitable foundations can also help direct funding to specific, related areas of research, thus avoiding duplication between funding agencies and maximizing progress in that area as a whole.

Furthermore, by ensuring that priorities are publicized effectively in scientific and clinical arenas, private foundations can increase overall awareness of the progress and issues involved, which will, in turn, influence other strands of funding (eg Government sources). Again, this further increases the speed and effectiveness of particular research directions. One example of the way in which funding from several organizations has been used to develop a successful avenue of research is provided by the use of the antiinflammatory agent methylprednisolone as a treatment to reduce damage in acute SCI. Several private foundations, as well as Government agencies, were involved in funding different aspects of the multicentre research programme that led to this important clinical advance (the NASCIS trials). ${ }^{1}$

\section{Supporting multidisciplinary approaches}

Another advantage of a goal-driven approach to funding scientific and medical research is that it enables private foundations to provide the impetus and support for basic researchers, clinicians and others to work together in ways that are not traditionally supported by Government sources. This is an important consideration as it becomes increasingly accepted that clinical treatment is likely to involve a combination of techniques, and it will become increasingly important as advances in basic research progress closer towards therapeutic application. Although it is vital that scientific advances in the laboratory progress as quickly as possible to clinical trials in human patients, the safety of the individuals concerned is paramount. Translational research, the process by which basic research advances are developed into useful clinical therapies, is not generally considered an attractive area of research for career scientists. This type of research is a complex, expensive, time-consuming process that receives little Government funding and, despite the potential benefits, the relatively small number of individuals affected by
SCI each year makes it unattractive to the major pharmaceutical companies. Undoubtedly, charitable foundations will play a crucial role in guiding and directing the translational studies that are legally required to assess the efficacy and safety of potential therapies before they can progress to trials in humans. Having the means to determine reliably the changes that result from any treatment in humans is an important additional factor in the move forward to this next stage of research.

\section{Increasing awareness}

Private foundations also have a further role in ensuring that the issues and priorities involved are publicized effectively to members of the general public and individuals living with SCI. This creates awareness of the human and financial costs associated with SCI and need for further research, which can be harnessed to influence other funding sources.

\section{International Campaign for Cures of Spinal Cord Injury Paralysis}

It is with the above issues in mind that a group of private foundations with similar aims came together in 1997 to form the International Campaign for Cures of Spinal Cord Injury Paralysis (ICCP; www.campaignforcure.org). ICCP members fund SCI research around the world, principally in Europe, the US, Canada and Australia. In the 2001 financial year, ICCP member groups funded over 150 basic and clinical research projects worth over US\$23 million.

Currently, ICCP members comprise:

Australasian Spinal Research Trust (ASRT), Australia; Christopher Reeve Paralysis Foundation (CRPF), USA; International Spinal Research Trust (ISRT), UK; Institut pour la Recherche sur la Moëlle Épinière (IRME), France; Kent Waldrep National Paralysis Foundation (KWNPF), USA; Miami Project to Cure Paralysis (MP), USA; Paralyzed Veterans of America (PVA), USA; Rick Hansen Institute (RHI), Canada; Spinal Research Fund of Australia (SRFA), Australia.

The mission of ICCP is to expedite the discovery of cures for SCI paralysis. By working together as a coherent group, member organizations will ensure that effective treatments that address issues relevant to people with SCI are developed as quickly as possible.

\section{ICCP objectives}

All ICCP member organizations subscribe to the following key objectives:

1. Attract the best and brightest scientists, researchers and clinicians to the field of central nervous system nerve regeneration and repair, particularly those who 
are newly graduating, and encourage their career commitment to spinal cord research.

2. Promote public support for the development of effective treatments and cures by highlighting the individual vulnerability to injury and the benefits of cures to present and future generations.

3. Promote government financial support for spinal cord research by highlighting the economic cost of lifetime care for those injured.

4. Give consideration to conducting collaborative awareness and fundraising campaigns to promote the global nature of spinal cord injury and paralysis cure research.

5. Promote linkages and communications between laboratories, scientists, clinicians and other relevant organizations.

6. Promote heightened communication between fundraising groups and encourage shared utilization of resources and expertise.

7. Encourage the development of strategies and priorities for the international field of spinal cord injury research.

8. Evaluate the progress and success of the campaign against concrete measurable outcomes and report progress to the field.

\section{ICCP achievements}

Quarterly telephone conferences, backed up by regular meetings of the members of ICCP provide a vital forum in which to discuss issues that are relevant to all members as well as opportunities to progress the above objectives.

Member organizations have collaborated to produce an information booklet that details the aims of ICCP and SCI research in terms that can be easily understood by the general public. This is updated annually to provide a valuable resource that can be used by individual member organizations for fundraising and educational purposes. In addition, ICCP maintains a website as a further information resource for individuals, the press, organizations and governments who wish to understand more about SCI research and obtain information on past and current funding provided by the members.

To further its mission to encourage and facilitate a lifelong commitment to the held of spinal cord repair, the ICCP has established an annual ICCP Outstanding Young Investigator Award.

By awarding two US\$3500 grants each year, ICCP intends to promote research to repair the damaged spinal cord, encourage young postdoctoral fellows to devote their careers to SCI research, and stimulate collaborative research. Awards are given on scientific merit according to the recommendations of an international scientific panel. In 2003, awards were given to Dr Valeria Cavalli from the University of California, San Diego and Dr Michele Lemons from the University of Utah, USA. Previous recipients were Elizabeth Coulson, The Walter and Elizabeth Hall Institute, Royal
Melbourne Hospital, Australia (2000), Jeffrey Petruska, SUNY Stony Brook, New York, USA (2001), Stephen Crocker, Neuroscience Research Institute, Ottawa, Canada (2001), Andrea Huber Brosamle, Johns Hopkins University School of Medicine, USA (2002) and Rodney L Rietze, The Walter and Elizabeth Hall Institute, Melbourne, Australia (2002).

\section{Neurotrauma initiatives}

As a result of pressure from private lobbyists and with the backing and advice of ICCP members, new funding initiatives have been developed in Australia, Canada and the USA, where state or provincial governments are able to raise or appropriate funds independently from federal politics. Typically, revenue is raised through road traffic offence fines and redirected towards neurotrauma research and road safety issues. This is leading towards an increasingly significant new source of funds, albeit in a local fashion, and has already affected the demographic spread of spinal cord injury research laboratories.

ICCP also encourages the work of additional organizations, particularly in countries that lack a tradition of charitable funding of medical research and to assist similar organizations with fewer resources. In the USA, input from ICCP members has helped raise the profile of SCI in general, which, indirectly, has helped to increase government funding for SCI research. New initiatives established by NINDS include the US\$8 million Facilities of Research Excellence in Spinal Cord Injuries (FORE-SCI) programme. This has four main objectives: setting up a new course to teach SCI methods; recruiting and providing new training opportunities for researchers entering the field of SCI; replication of some acute and chronic injury studies; and development of new behavioural tests to assess recovery from SCI.

This programme is unique in specifically addressing one of the major issues associated with translating any experimental observations to a potential clinical treatment, that of reproducibility. Before proceeding to clinical trials with confidence, the success of experimental treatments must be confirmed - this applies to all medical research, not just to SCI. It is likely that many of the apparent difficulties in reproducing some of the promising SCI results are technical. It is inevitable that different laboratories favour slightly different models and ways to assess the effects of treatment, and these are likely to lead to apparently conflicting results. ${ }^{2}$ Providing the incentive to confirm results in independent studies (in consultation with the original authors) should overcome this, and strengthen the basis on which experimental studies are translated into effective treatments for SCI injury. These initiatives can only be of benefit to the future of the field.

\section{Clinical trials guidelines}

ICCP members are also committed to establishing guidelines that will enable reliable clinical trials of 
treatments for SCI that will be accepted by scientists worldwide. Already, a small number of 'trials' of potential treatments have received publicity, despite the fact that clear, objective and accurate methods of measuring and monitoring the status and severity of an SCI lesion (and, therefore, improvements following treatment) are yet to be developed. In addition, such trials have gone ahead in isolation without evidence of reproducibility by other laboratories. Unfortunately, premature trials run the risk of discrediting the whole of this area of research, in much the same way that gene therapy trials have received recent setbacks. To prevent this, ICCP members are working together to establish guidelines for clinical trials that are accepted by member organizations and can be used as a benchmark to guarantee the quality of future research. By adhering to these guidelines, individuals with SCI who are considering enroling in trials, researchers who are involved in organizing and assessing trials and the funding organizations themselves will be guaranteed that the study conforms to meaningful guidelines, outcome measures and safety considerations.

\section{ICCP affiliate organizations}

Although the organizations are all working toward the same goals, they differ in their individual approach according to their charitable remit, income and philosophy. The descriptions below indicate their general strategies and more specific information can be found at each of the organizations' websites.

\section{Australasian Spinal Research Trust (ASRT)}

The ASRT (www.spinetrust.com.au) was founded in 1994 by Stewart Yesner with the mission to raise funds for research to expedite a cure for the paralysis caused by SCI. Currently, the major proportion of income $(\sim 40 \%)$ comes from government sources, with the remainder divided among corporate sources $(\sim 20 \%)$, individual members $(\sim 20 \%)$, sponsorship $(\sim 10 \%)$ and special events $(\sim 10 \%)$.

The ASRT advertises for grant applications, which are reviewed and prioritized for funding by a Scientific Committee. In general, successful applications receive 1 year's funding, which can be extended. In addition, the ASRT has limited funding to sponsor the attendance of leading researchers at conferences that focus on cures for spinal cord injury.

Recent projects for which ASRT has provided partial funding include investigations of adult stem cells at Walter and Eliza Hall Institute for Medical Research under the control of Professor Perry Bartlett and Professor Phil Waite's work on olfactory tissue at the University of New South Wales.

\section{Christopher Reeve Paralysis Foundation (CRPF)}

CRPF (www.christopherreeve.org) is a national, nonprofit organization in the US that is committed to funding research that develops treatments and cures for paralysis caused by SCI and other central nervous system disorders. The Foundation also works vigorously to improve the quality of life for people living with disabilities through the Christopher and Dana Reeve Paralysis Resource Center, a Quality of Life grants programme, and advocacy efforts. CRPF was formed in 1999 by the merger of the American Paralysis Association (APA), and the Christopher Reeve Foundation (CRF). Established in 1982, the APA itself brought together several small family foundations formed after a loved one's SCI. Christopher Reeve and his wife Dana formed the CRF to raise funds to establish a Quality-ofLife grants programme and support the APA's research programmes after the equestrian injury in May 1995 that rendered him a C1-2 ventilator-dependent tetraplegic.

CRPF funding comes from individuals (47\%), corporations and foundations $(16.6 \%)$, special events $(35 \%)$ and federated giving campaigns, etc. (1.4\%). The majority of resources are invested in research programmes, with US\$7,390,000 budgeted for 2003. The Quality-of-Life grants programme has an annual budget of US\$700,000 for selected disability organizations worldwide.

CRPF supports spinal cord research in laboratories around the world through two funding programmes. The Individual Research Grants programme awards 2year grants (US\$75,000 maximum per year) to senior scientists, young investigators and postdoctoral fellows who are selected using councils of internationally renowned neuroscientists and clinicians. Postdoctoral fellowships are also available with a maximum funding of $\$ 60,000$ per year for 2 years. CRPF is presently organizing an inaugural March 2004 grant holders' meeting to be held every other year. The Foundation also supports a Research Consortium on Spinal Cord Injury, which has seven member laboratories (six in the US and one in Zurich, Switzerland), each funded for 3year periods (US\$260,000 per laboratory per year). The Consortium programme also funds an animal core laboratory facility at the University of California, Irvine (US\$455,000 per year) and a microarray core laboratory facility at The Salk Institute for Biological Studies, La Jolla, CA (US\$200,000 per year).

Presently, CRPF research awards focus on limiting the initial damage after injury, preventing secondary neuronal cell death, promoting regeneration and remyelination and improving secondary complications such as bowel, bladder and sexual dysfunction, pain and spasticity. Historically, the majority of grant funding has been at the basic end of the research continuum, with emphasis on the biology of spinal cord repair. However, as research advances there has been a significant shift towards supporting clinical research and CRPF anticipates launching translational research initiatives in 2003.

\section{Institut Pour la Recherche sur la Moëlle Épinière (IRME)}

IRME (www.irme.org) is a private nonprofit organization founded in 1984 at the initiative of Jean Delourme, 
grandfather of a tetraplegic paralysed after a road accident when aged 20. Its aim is to contribute to the understanding of spinal cord lesions by promoting and supporting research on spinal cord injury repair in France. A 14-member Board of Trustees sets the policy of the organization and raises the funds for the research programmes. An international Scientific Advisory Board composed or 14 basic scientists and clinicians evaluates the applications submitted by the research laboratories and provides the Board of Trustees with comments, recommendations and proposals for grants.

The annual income is over $€ 500,000$, from private donations and voluntary fundraising efforts. In the period 1995-2000, €2,439,000 was allocated to research.

The IRME strategy is to found a network of basic research laboratories and clinical departments with the objective to develop therapeutic strategies for spinal cord trauma. IRME presently supports and coordinates the work of seven basic laboratories and clinical departments involved with spinal cord injury repair, all linked with French public institutions such as the CNRS (National Council for Scientific Research), INSERM (National Institute for Medical Research) or various Universities.

Four main research lines are being followed:

(1) Preventing the spread of secondary lesions

(2) Control of the glial scar and axonal regrowth

(3) Restoration of spinal cord function using neural grafts

(4) Cellular and gene therapy

Since 1997, IRME has organized regular international meetings, including three major conferences at Deauville on Spinal Cord Injury.

IRME is also involved, together with other organizations, in a new multicentre study on improving and standardizing the emergency care system for spinal cordinjured people.

\section{International Spinal Research Trust (ISRT)}

ISRT (www.spinal-research.org) is a UK-based medical research charity founded by Stewart Yesner in 1980 with the sole remit of funding research aimed at alleviating the effects of paralysis caused by spinal cord injury.

The Trust receives no government funding and in recent years has relied heavily on targeted approaches to charitable trusts for a significant section of its income. During FY 2002-2003, the relative proportions of income were: general fundraising and events $57 \%$, Trusts $21 \%$, investments $13 \%$ and other sources $9 \%$. Annual expenditure has risen to approximately $£ 1.5 \mathrm{~m}$, funding projects based in the UK, Europe, Canada and the USA.

Following publication of the first Strategy for Research in $1996,{ }^{3}$ ISRT funding has been targeted towards areas of research that warrant specific attention or would not be easily supported by mainstream granting agencies. The Strategy is continually reevaluated and an updated version appeared as a major review of the field in 2000. ${ }^{4}$ Funding is mainly international, in the form of competitive 3-year project grants to support postdoctoral researchers in the basic sciences. Occasionally, projects are commissioned if there is adequate justification. In addition the Nathalie Rose Barr PhD studentship scheme, for 3- or 4-year projects designed to encourage young investigators to undertake a career in SCI research, has so far led to the support of 17 research students. All projects are awarded following peer review by a panel of internationally renowned scientists.

Current projects extend across a wide range of topics: investigating and controlling the early phases of inflammation, elucidating the mechanisms behind the regenerative capacities of olfactory glia, improving the function of partially damaged axonal fibres, to understanding the intrinsic pathways responsible for the control of locomotion. A major multidisciplinary programme, the Clinical Initiative, is underway, aimed at perfecting standardized assessment protocols that are required to provide a sound framework before clinical trials of potential therapies can be initiated with any degree of reliability. 5

The Trust holds an annual meeting of those working on all its grants, to encourage debate and collaboration as well as forum where specific bottlenecks to progressing the field can be identified and prioritized, hence influencing future funding initiatives.

Kent Waldrep National Paralysis Foundation (KWNPF) Kent Waldrep formed the KWNPF (www.spinalvictory.org) in 1985 to support research that will unravel the mysteries of spinal cord regeneration. Fundraising efforts have focused on building a state-of-the-art paralysis research centre at the University of Texas Southwestern Medical Centre in Dallas, USA. This has culminated in a US\$15 million basic research endowment that supports Dr Luis Parada and some 60 scientists to develop genetic engineering and clinical therapies that address regeneration and better functional outcomes. For example, one of the priorities is a treadmill-training programme.

The KWNPF uses a small science advisory board to review priorities and approve grant funding. Its policy is to seek out the science that they wish to support and target funding on those few projects. Although complimentary medicine is not supported, it is not discouraged, as many forms of complimentary medicine (including chiropractic care, for example) might benefit the spinal cord-injured community.

About US\$2 million in research is normally supported each year, with US\$1.5 million earmarked for UT Southwestern Medical Centre. If funds were sufficient, the centre would be extended to include a clinical research and rehabilitation facility that would coordinate with other major research centres around the 
world. Transplant and stem cell research would also be pushed forward to develop therapies that might reverse paralysis in some incomplete injuries. Another priority is to continue to support symposiums that promote collaborations and better interaction between the basic science community and clinicians.

There is an underlying opinion at KWNPF that in this decade, treatments will be developed that will help improve the quality of life for many people with SCI. Although it may never be possible to restore $100 \%$ of function, alleviating pain and spasticity and return of bladder function would allow many a quality of life that they never thought they would enjoy. Return of movement will happen at some point in the nature: money and leadership in the scientific community will affect this time table.

\section{The Miami Project}

The Miami Project (www.themiamiproject.org) follows a slightly different model from other member groups in that, rather than making external awards, it funds research undertaken by a multidisciplinary group of scientists located under one roof in what is designated a Centre of Excellence at the University of Miami School of Medicine.

The Miami Project was founded in 1985 by Barth Green, MD, a surgeon with an interest in scientific research, and three equally committed individuals, Don Misner, Beth Roscoe and Nick Buoniconti, who had experienced spinal cord injury either themselves or in a close relative. They envisioned that a group of scientists in the same location, each bringing different expertise to the problem of spinal cord repair, would enjoy greater success than a more fragmented approach.

Today, the Miami Project is a multidisciplinary research centre that remains committed to this original vision. The major proportion $(\sim 70 \%)$ of the research undertaken involves basic science. The remaining $30 \%$ is composed of clinical and rehabilitation studies, areas that would be increased should more funds become available. In addition, the Miami Project supports educational activities in what is a 'holistic' approach to SCI.

Around $50 \%$ of the annual budget of the Miami Project comes from grants and contracts awarded to individual investigators; individuals, corporations and private foundations provide the remainder. Annual reports on individual scientific programmes are available and funding decisions are based on productivity and scientific importance.

A primary goal of the Miami Project is to increase the number of scientists working on spinal cord injury. To this end, fellowships established by private donors are available to support trainees (graduate students and post-doctoral fellows) at the Miami Project and the University of Miami Neuroscience Program. The Miami Project also receives funding from the NINDS-sponsored Facility of Research Excellence for Spinal Cord Injury Programme to train summer students, postdoctoral fellows and visiting scholars.
Some of the major advances that involve members of the Miami Project include the development of hypothermia, IL-10 and gangliosides as promising neuroprotective strategies in acute SCI. Members of the Miami Project are also involved in the use of Schwann cells, olfactory ensheathing glia, neurotrophins and stem cells to aid regeneration in chronically injured animals.

One example of the way in which research has already impacted on the day-to-day clinical management of people with SCI is the increased clinical awareness of the detrimental effects of secondary insults, such as hyperthermia and hypoxia, which occur following SCI and the measures that are now being taken to minimize these insults. In chronically injured individuals, advances in rehabilitation and exercise training that have led to improvements in health have made an impact on the philosophy of rehabilitation following SCI.

\section{Paralysed Veterans of America (PVA)}

PVA (www.pva.org) was founded in 1947 to help the 2000 American Servicemen and women who suffered spinal cord injuries during World War II. One of its first goals, and still part of its mission statement, is to fund quality research to help people with spinal cord injury and disease.

The Technology and Research Foundation (later renamed Spinal Cord Research Foundation, SCRF) of the PVA was established in 1975 with the aim of alleviating and eventually ending the medical consequences of paralysis, improving the current treatment and care for spinal cord dysfunction, developing innovative rehabilitation therapies and assistive devices and training the next generation of researchers. SCRF allocates funding using a system in which the Board of Trustees, which consists of PVA members (veterans with a spinal cord injury or disease) and staff, vote on the grants assisted by a scientific review committee who help to interpret the research proposals. This allows a consumer group to fund grants that will make a difference to their lives, based on the recommendations of expert scientist. As far as we are aware, no other spinal cord injury foundation operates tins way.

Since its interception, SCRF has provided more than US $\$ 30,000,000$ to laboratories and hospitals in the US and Canada, of which $\sim$ US\$14,000,000 has funded basic research and $\sim$ US\$6,000,000 clinical research.

SCRF provides 2-year grants in the following areas:

1. Basic sciences related to spinal cord injury or disease, with the goal of finding a cure;

2. Clinical and functional studies of the medical, psychosocial and economic effects of spinal cord dysfunction, as well as interventions proposed to alleviate these effects;

3. Design and development of new and improved rehabilitation and assistive devices for the spinalcord dysfunctional populations;

4. Fellowships for postdoctoral scientists, clinicians and engineers to encourage the training and specialization of individuals in spinal cord research; 
5. Conferences and symposia that provide opportunities for interactions among scientists, health-service providers and other professionals involved with paralysis.

Most categories are awarded US\$75,000 per year: fellowships receive US $\$ 50,000$ per year for up to 2 years; conferences are usually awarded US\$2,000-15,000.

Realizing the importance of clinical science, PVA has targeted specific areas of clinical research in a directed research process. The amount allocated for such research might increase in the next few years as a result of increased awareness of areas where there are gaps in basic, clinical and technological research.

Financial support for grants is provided primarily by PVA, including all staff salaries. Recently, SCRF has begun to receive more funding from additional sources, including large donations from individuals who contribute through payroll deduction, gifts, wills, bequests, as well as a significant amount from PVA chapters. It hopes to diversify funding further by increases in individual donation and new partnerships with corporations and foundations. With a diversified income stream, SCRF will be able to increase the funds it awards each cycle with hopes that therapies will be available in the near future.

\section{Rick Hansen Man In Motion Foundation (RHMIMF)}

Rick Hansen came to the world's attention when, staring in 1985, he wheeled around the world in a journey lasting 2 years, 2 months and 2 days. He raised over C\$26 million for SCI research, rehabilitation and wheelchair sport, and created the RHMIMF (www.rickhansen.com).

The Rick Hansen Spinal Cord Injury Network (RHSCI-Net) brings together three diverse communities:

- Researchers - to accelerate discoveries and their subsequent translation into new treatments and cures.

- SCI service providers - to meet rehabilitation more effectively, wellness, housing and community programme needs.

- People with SCI, their family and friends - to articulate needs and interests, and to influence research and service programmes.

A central spinal cord registry and database is being created to enable these groups to interact and share information. To date, the RHMIMF has provided over C\$148 million and Rick Hansen is committed to building on this. His determination, leadership and vision are the driving forces behind the collaborative approach that brings these groups together with government, nongovernmental organizations, scientists, philanthropists and the private sector, to accelerate improvements to the quality of life for people with spinal cord injury.

The following programmes form the RHSCI-Net.
International Collaboration of Repair Discoveries (ICORD) ICORD is the hub of a research network that strategically links researchers to set common goals and share information, and to allow fast and accurate translation of basic discoveries into new drug and rehabilitation therapies biotechnical devices and surgical practices. Current projects include: building capacity and networking through retention and support of researchers; creating an interactive, web-based, on-line registry for patients with SCI; sexual and reproductive health research; rehabilitation strategies to improve the quality and length of life by examining the effectiveness of traditional/current interventions in SCI; and social research.

ICORD will be housed in a new international research centre that incorporates discovery research with clinical and rehabilitation research and practices. The facility is scheduled for completion in 2005. A partnership between the RHMIMF, the University of British Columbia and Vancouver Coastal Health Authority, this new research centre will provide equipment and facilities for over 300 researchers and 12 visiting research teams that focus on minimizing the effects of the spinal cord injury to prevent paralysis, maximizing physical function for people living with SCI and regenerating the spinal cord to restore full physical function.

Rick Hansen Neurotrauma Initiative (RHNI) Created in 1997 to mark the 10th anniversary of the Man In Motion World Tour, this national programme was designed to increase support and respond to the needs of people who survive brain and spinal cord injuries, and to fund research, rehabilitation and injury prevention in programmes. It continues as the RHNI in British Columbia and as tine Ontario Neurotrauma Fund in Ontario.

Acknowledging that the number and severity of brain and SCIs and that driving offences add to the risk of neurotrauma, the $\mathrm{BC}$ Government directs a portion of the provincial traffic offences to the field, through RHNI. Annually, C\$2 million is directed towards research as well as to the community through the Brain Injury Associations of $\mathrm{BC}$ and the $\mathrm{BC}$ Paraplegic Association to support neurotrauma rehabilitation and injury prevention initiatives.

With the current focus on ICORD and other research initiatives, no external applications are being accepted through the RHMIMF, the Rick Hansen Institute, The RHNI-BC or the Canadian Neurotrauma Research Programme. All grants currently funded under these programmes will continue.

Spinal Research Fund of Australia Incorporated (SRFA) Dawn Ferrett and Neil Sachse founded SRFA (www.sfra.com.au) in 1994 to exploit an opportunity to gain significant funds for research into treatment for SCI. An independent review identified that successful 
treatment for SCI had the potential to reduce the costs of insurance claims. High-profile business people were sought to form the SRFA Committee and a business plan was developed in which Corporate Sponsorship (from, for example, the Insurance Industry) provides the funds to support research projects and public support covers administrative costs. More recently, as the focus has narrowed to concentrate on research that will lead to clinical trials; a greater source of fundraising has to be unearthed to fund this.

SRFA supports research directed towards developing treatments, as distinct from basic research. Rather than advertising for research proposals, projects are first identified and, subsequently, sponsors are approached for funding. This is limited to projects in Australia, with the quality of research ensured by external review by national and international experts.

Professor Robert Rush, an expert in growth factors working at Flinders University, Adelaide has been funded for 7 years to develop a treatment to reverse paralysis in humans. A mid-term, independent review by scientific and clinical experts recommended that this project be continued. In addition, submission of a patent application was considered as a method to encourage further development of this treatment by commercial entities. This research project has had considerable success and resulted in publication of two significant articles, one on stimulating nerve fibres to grow past the site of injury, and the other a novel method to identify new nerve growth. SRFA wishes to progress these to a clinical trial in chronic SCI patients.

SRFA also identified and funded a second, 1-year project at the Garvan Institute, Sydney to develop a system of delivering neurotrophin genes directly to motor neurones in the corticospinal tract to stimulate regeneration and restore functional connections.

Both projects have encouraged new researchers into the field as well as making others aware of the progress in spinal injury research. SRFA also understands that developing a spinal injury treatment requires many different expertises and endorses a research-network approach as the best method to fulfil this aim. SRFA joined the ICCP to share information in an attempt to accelerate prospects of new treatments in clinical practices.

\section{Note added in proof}

After 18 years, The Kent Waldrep National Paralysis Foundation closed at the end of July 2003. The organization raised over US\$21 million for SCI research during this period.

\section{Acknowledgements}

We thank the past and present staff of all the ICCP member organizations for providing much of the information used in this article.

\section{References}

1 Bracken MB et al. A randomized, controlled trial of methlprednisolone or naloxone in the treatment of acute spinal cord injury. $N$ Engl J Med 1990; 322: 1405-1411.

2 Pearson H. In search of a miracle. Nature 2003; 423: 112 113.

3 Harper GP, Banyard PJ, Sharpe PC. The International Spinal Research Trust's strategic approach to the development of treatments for the repair of spinal cord injury. Spinal Cord 1996; 34: 449-459.

4 Ramer MS, Harper GP, Bradbury EJ. Progress in Spinal Cord Research: a refined strategy for the International Spinal Research Trust. Spinal Cord 2000; 38: 449-472.

5 Ellaway $\mathrm{PH}$ et al. Towards improved clinical and physiological assessments of recovery in spinal cord injury: a clinical initiative. Spinal Cord 2004, In press. 